\title{
Industrial robot trajectory optimization- a review
}

\author{
Mariana Ratiu ${ }^{1 *}$, Mariana Adriana Prichici $^{2}$ \\ ${ }^{1}$ University of Oradea, e-mail: mratiu@uoradea.ro, Romania \\ ${ }^{2}$ University of Oradea, e-mail: mprichici@uoradea.ro, Romania
}

\begin{abstract}
This paper is the result of the literature review on the trajectory optimization of the serial industrial robots, a study developed in a wider research in the field of the trajectory generation mechanisms of the serial industrial robots. After a short presentation of the importance of the industrial robots in the current context and future challenges, are presented the main optimal trajectory planning criteria approached in several specialized scientific papers, especially in the last few years.
\end{abstract}

\section{Introduction: Current Context}

In one of the many definitions of the industrial robots in ISO $8373: 2012$ [1] is said that an industrial robot is an "actuated mechanism programmable in two or more axes with a degree of autonomy, moving within its environment, to perform intended tasks". There are different types and models of industrial robots, which can be classified, as M. W. Spong, S. Hutchinson and M. Vidyasagar [2] say, according to different criteria, such as the power source or the way in which their joints are actuated, their mechanical or kinematic structure, the payload capacity, the volume of their workspace, their method of control or their intended application area.

Current and future challenges to effectively respond to the global competitiveness and the consumer behaviour, and using the advantages of the new technologies, aim, as M. Hägele, K. Nilsson, N. Pires and R. Bischoff say [3], to design the industrial robots after new principles, so they can be used in many fields and industries, to be more performance and less expensive, to interact intuitively with workers. H. Chen, B. Zhang and G. Zhang [4] note that such intelligent industrial robotics systems are attracting more and more attention of the specialists because of the growing need for adaptation to the complex and flexible industrial processes. C. Mineo, S. G. Pierce, P. I. Nicholson and I. Cooper [5] prove that automatic programming of control systems based on robots does increase flexibility by minimizing the effort and time needed for implementation.

R. Bloss [6] shows that a class of robots that successfully meet such challenges is the category of the collaborative robots, which can be used in many applications where traditional robots fail, providing rapid major improvements in productivity, safety, ease of programming, portability and costs over time. R. Bogue [7] and B. Carlisle [8] take into account the use of the collaborative robots in applications such as the manufacture and assembly of the electronic products or those from the automotive; they can be used by the small and medium-sized companies or by the companies seeking agile production methods. Z. Lu, C. Xu, Q. Pan, D. Xiao, F. Meng and J. Hao [9] analyse the use of the collaborative robots for non-destructive testing of curved surfaces. O. Madsen, S. Bogh, C. Schou, R. S. Andersen, J. S. Damgaard, M. R. Pedersen and V. Krüger [10] present the integration of two autonomous mobile robots in a real manufacturing environment.

It is expected to expand to new areas the applicability of the industrial robots, such as deburring of custom parts with complex geometry, high-speed processing, such as milling with a high degree of accuracy, as A. Klimchik, A. Ambiehl, S. Garnier, B. Furet and A. Pashkevich [11] present, or arc welding. J. Léger and J. Angeles [12] say that all these require high precision robots, robots whose components are built and assembled with precision and the trajectory planning and control algorithms are also designed to improve the accuracy execution of the tasks. T. Messay, R. Ordóñez and E. Marcil [13] note that an improvement of the accuracy of the robot can be obtained by a computationally efficient and kinematic calibration method.

According to G. Berselli, M. Gadaleta, A. Genovesi, M. Pellicciari, M. Peruzzini and R. Razzoli [14], the industrial robots could be an effective alternative to the dedicated machine tools, because of the increased

\footnotetext{
"Corresponding author: mratiu@uoradea.ro
} 
flexibility of the possibility of location and cost, being estimated a reduction of $20-50 \%$ costs compared to the machining with the traditional CNC machines. To ensure efficiency of the entire robotic system in the production processes, the researchers seek, study and propose various machining strategies and robot settings leading to improved geometric accuracy and surface quality processed using industrial robots, M. Halbauer, C. Lehmann, J. P. Städter, F. Leali [15], the robot stability to the vibrations occurring in the machining process (chatter), S. Mousavi, V. Gagnol, B. C. Bouzgarrou, P. Ray [16].

In the context of the extensive use of the industrial robots in automated production processes, P. Tavares, J. Lima, P. Costa and A. P. Moreira [17] seek the best solutions to optimize and streamline the entire process. In this regard, G. Boschetti [18] proposes and experimentally implements a path planning technique for a robot so that it can pick-up parts "on the fly" of the circular feeder without to need to stop its rotation. To overcome the problems of the occurrence of the equipment failures, C. Müller, C. Weckenborg, M. Grunewald and T. S. Spengler [19] propose taking into consideration of the redundancy in the configuration of the automated assembly lines, by creating a buffer space between the stations, so that the downstream stations to take over the operations of the failed stations.

Nowadays, when it is imperative to develop environmentally friendly products and production processes, many studies in robotics are conducted regarding the reduction of the energy consumption, in special through the optimization of the robot's motions, as L. Wang [20] says. D. Constantinescu and E. Croft [21] demonstrate that the optimization through the smoothing trajectories provides a superior feasible optimal time and leads to the reduced energy consumption, as Paryanto, M. Brossog, M. Bornschlegl and J. Franke [22] show. To ensure a sustainable manufacturing, recently was appeared the concept "Industrial Cloud Robotics" presented by J. Liu, W. Xu, J. Zhang, Z. Zhou and D. Pham [23].

P. Tavares, J. Lima and P. Costa [24] propose a solution for the need to adapt the robots at the multiple loads and environments by creating a Robotic Operative System (ROS), a set of software libraries and tools that can be used for building robot applications by putting together more simple algorithms, in order to obtain a modular solution to a complex problem, thus increased flexibility. There are also available open-source educational platforms, with robots with different degrees of freedom, which can provide academic training to those interested without charge, as S. Manzoor, R. Ul Islam, A. Khalid, A. Samad and J. Iqbal [25] show.

Considering the role of the robots in the present and future industrial processes, a lot of recent studies are conducted to streamline and optimize the trajectories and the motions of the industrial robots.

\section{Industrial robot trajectory optimization}

Optimization of the movement of an object manipulated by an industrial robot, between two given positions, involve both optimization of the movement of the object manipulated respectively of the end-effector relative to the robot base, as well as the optimization of the positioning of the robot base relative to the application defined by the two positions, or vice versa, the positioning of the application relative to the robot base. D. Spensieri, J. S. Carlson, R. Bohlin, J. Kressin and J. Shi [26] demonstrate experimentally that the optimal position of the robot base into a cell assembly in automotive, depending on the tasks to be performed, leads to a reduction of up to $20 \%$ of the execution time of the tasks.

The purpose of such optimization is to minimize/maximize at least one of the following objective functions:

1) minimizing the execution time, respectively maximizing the robot productivity, considering that the relative speeds of the actuator's elements are limited constructively;

2) minimizing the energy consumption or mechanical work necessary for execution, leading to a reduction of the mechanical stresses in actuators and on the robot structure and obtaining smooth trajectories, easy to follow;

3) minimizing the maximum power required for operating the robot;

4) minimizing the maximum actuation forces and moments.

Often, to get better results, multi-criteria optimization is achieved when the objective function that minimizes/maximizes express simultaneously more of the above criteria.

The most common optimization criteria used in the literature are: minimum time trajectory planning; minimum energy trajectory planning or minimum actuation effort; minimum jerk trajectory planning, or hybrids criteria, such as, for example, minimum time and minimum energy, when is a compromise between the time necessary for trajectory and the energy of the actuators; minimum time and minimum jerk, both minimized simultaneously.

J. Kim and E. A. Croft [27], following a study in terms of the industrial applications of the available optimization methods, claime that these methods are limited to use, if not taken into account some important considerations such as the precision tracking trajectory, the importance of the minimum time criteria, the need to minimize the cycle time by online planning, the motion controllers' performance.

\section{Minimum time trajectory planning}

Minimum/optimal time trajectory planning is the first and most frequently method of optimization discussed in the literature, especially due to its direct connection with the reduction of the manufacturing time respectively with the productivity increase. Over time, have been used

* Corresponding author: mratiu@uoradea.ro 
various techniques or different algorithms for this type of optimization but most of them neglect the actuator dynamics, which lead to discontinuation of the actuators' accelerations and torques. To overcome this disadvantage, D. Constantinescu and E. Croft [21] solve the optimization problem by using a flexible tolerance method and limits for the torque rates to obtain the desired smoothness for trajectory which provides a feasible time-optimal motion. X. Gao, Y. Mu and Y. Gao [28] use for optimization an improved teaching-learning algorithm while respecting the kinematic constraints. J. Kim, S. R. Kim, S. J. Kim and D. H. Kim [29] recommend an approach for online implementation by using the dynamic model of the robot to find the maximum kinematic constraints that are used with the conventional trajectory models. F. Abu-Dakka, I. Assad, R. Alkhdour and M. Abderahim [30], after an exhaustive analysis of the optimization methods presented in the literature, prefer the optimization by minimizing the time, using a parallel populations genetic algorithm and three different types of constraints: kinematics, dynamics and payload constraint, instead of minimizing the jerk, the latter being placed constraints within the meaning of imposing limits given in the literature.

In the context in which was found that the redundant robots have some advantages in comparison with the conventional robots, there are numerous studies on optimizing their motions. A. Reiter, K. Springer, H. Gattringer and A. Müller [31] present a method that uses, besides of the standard optimization techniques, a decomposition of the joint space and inverse kinematics analysis for obtaining a minimum-time trajectory of the joint. G. Erdős, A. Kovács and J. Váncza [32] propose the optimization method for a redundant industrial robot that execute a set of tasks in a complex work environment with multiple technological and geometric constraints, consists of selecting, from the set of configurations obtained by solving inverse kinematics, of those which avoid collisions, after which, pursuing the time minimization by ensuring the shortest trajectory, is selected the optimal combination, respecting all kinematic constraints of the robot.

Starting from the observation that only a few studies approach the working times in direct connection with the analysis of the productivity, F. Rubio, C. Llopis-Albert, F. Valero and J. L. Suñer [33] demonstrate, by using two methodologies, an optimization procedure, and a simulation technique, that the optimization procedure, with multiobjective functions, leads to lower working times and greater annual revenue in comparison with those obtained with a simulation technique.

\section{Minimum energy trajectory planning}

The trajectory optimization by minimizing the energy has several advantages. On the one hand, it produces smooth trajectories that are easier to follow and reduces mechanical stresses in actuators and on the robot structure. On the other hand, this method saves energy, which is important not only regarding the cost but also regarding the quantitative limitation, in the case of specific applications where the power supply is limited by technical factors such as robotic applications in outer space or underwater exploration or for certain military tasks.

This type of optimization, developed in recent times in industrial applications, due to the need to reduce the energy consumption, is found in several studies: $T$. Mashimo, T. Urakubo and T. Kanade [34]; D. Meike, M. Pellicciari and G. Berselli [35]; O. Wigstrom, B. Lennartson, A. Vergnano and C. Breitholtz [36]; J. Gregory, A. Olivares and E. Staffetti [37]". In these studies are used different algorithms or methods for trajectory planning, including modeling and simulation. In some of these studies it was not succeeded obtaining the continuous accelerations and torques in joints, but in other ones, this inconvenience has removed. In one such study, L. P. Luo, C. Yuan, R. J. Yan, Q. Yuan, J. Wu, K. S. Shin and C. S. Han [38], by using the Lagrange interpolation method and through imposing position and stability constraints were obtained the smoothness of the joint angular positions, velocities, accelerations, and joint torques. R. F. Fung and Y. H. Cheng [39] use genetic algorithms and high-degree polynomials to optimize, by minimizing energy, the point-to-point trajectory of a robot, introducing constraints of the angular displacement, speed, acceleration, and jerk at the start and end times. M. Jin and $\mathrm{D}$. $\mathrm{Wu}$ [40], in a concrete study on a manipulator redundant with six degrees of freedom, to ensure the safety motions and to reduce the energy consumption, it was adopted an improved algorithm type "particle swarm optimization" (PSO) for optimizing the trajectory of the arm system.

Starting from the finding that the position-controlled servo systems, widely used and efficient, are energy intensive, G. Berselli, F. Balugani, M. Pellicciari and M. Gadaleta [41] demonstrate that the trajectory optimization by minimizing the energy leads to the best performance of these servo systems. Other approaches in this regard are found in several other recent studies: E. Oliva, G. Berselli, M. Pellicciari and A. Andrisano [42]; M. Pellicciari, G. Berselli and F. Balugani [43]; Y. Wang, K. Ueda and S. Bortoff [44]; M. Huang, Y. Hsu and R. Fung [45].

\section{Minimum jerk trajectory planning}

Jerk is the third derivative of the position in time and indicates how quickly the joints change its forces. Jerk's reduction ensures the trajectory continuity and leads, on the one hand, to the reduction of the joint positioning errors or more accurately tracking trajectory and, on the other hand, to the reduction of the robot vibration limits, which leads to the wear reduction and thus to the increasing the duration of the exploitation.

The trajectory planning through jerk minimization is performed in specialized researches by two approaches: by using predefined via points, called nodes, node points or control points used for interpolation and without the use of node points. H. I. Lin [46] presents a quick and unified method for joints' trajectory planning, by minimizing the jerk, using the PSO algorithm integrated

$\overline{\text { *Corresponding author: mratiu@uoradea.ro }}$ 
with the K-means clustering. Using a computer simulation method has been proved that this method is easy and efficient in comparison with other methods taken into consideration.

\section{Multi-criteria optimization}

Z. Miljković and M. Petrović [47] present, in an optimization study regarding the planning of a flexible manufacturing process, a hybrid optimization criterion, minimum production time and minimum production cost, by using an improved particle swarm optimization algorithm, with crossover, mutation and shift operator.

By applying the combination minimum time and minimum energy, which is a compromise between the time necessary for trajectory and the energy of the actuators, D. Gleeson, S. Bjorkenstam, R. Bohlin, J. S. Carlson and B. Lennartson [48] show that can achieve a $10 \%$ reduction in energy consumption of an industrial robot standard used in a welding station.

The hybrid optimization criterion minimum time and continuous jerk is applied in a study by H. Liu, X. Lai and $\mathrm{W} . \mathrm{Wu}$ [49] for obtaining efficient and smooth motions for the robot. In another studies by the same type, the criterion applied is: time-optimal and jerkbounded for a pick and place operation, by S. Perumaal and N. Jawahar [50]; time-jerk optimal, taking into account more constraints: the joints' velocity, acceleration and jerk by A. Gasparetto and V. Zanotto [51], A. Gasparetto, A. Lanzutti, R. Vidoni and V. Zanotto [52], and the heavy payload and high speed handling, F. Liu, F. Lin [53].

To optimize the trajectory of a glass-handing robot, in a study H. Duan, R. Zhang, F. Yu, J. Gao and Y. Chen [54] present a multi-criteria approach, through weighting the execution time, acceleration, and jerk, and the results of the mathematical simulations show not only improving the working efficiency but also ensuring smoothing robot' motions.

By using for optimization the objective functions minimum transmission time and maximum load carried, H. Esfandiar and M. H. Korayem [55] have planned an optimal point to point trajectory for a flexible manipulator under large deformation, by imposing kinematic constraints (maximum velocity and acceleration), dynamic constraint (maximum torque applied to the arms) and also the constraint of final point accuracy.

To increase flexibility and manoeuvrability, are sometimes used snake robots, which have a high degree of freedom, which prevents their control, but facilitates their motions on uneven surfaces. There are several studies that seek the best algorithms for the motion control of the joints of the snake robots, as M. Jafari and A. Shahmansoorian [56] show. In one such study, P. Costa, J. Lima, A. I. Pereira, P. Costa, A. Pinto [57]" propose a multi-criteria optimization for a robot with 12 degrees of freedom, Tentacle Robot, by minimizing the Euclidean distance between the target positions and by minimizing the effort of the servomotors.
To perform a complex robotic assembly task was developed by F. Anton, S. Anton, S. Răileanu and T. Borangiu [58] an algorithm based on the dynamic equations of the robot, to obtain the optimal speedtrajectory length, taking into account the load of the robot and the stopping points on the trajectory.

In an attempt to solve the kinematic redundancy of a $6 \mathrm{R}$ industrial robot used for five axes milling process, $\mathrm{W}$. Xiao, J. Huan [59] propose a numerical multi-criteria method to optimize the singularities, joint limits and collisions, critical constraints for the milling process.

To planning the trajectory of industrial robots with payload constraint, into a research S. S. Chiddarwar and N. R. Babu [60] impose six objective functions: weighted balance of traveling time, actuator torques, singularities avoidance, joint jerks, joint accelerations and gripping force.

\section{Optimization of the robotic task sequence}

In many industrial applications, the robots are used to perform a set of tasks and the tasks sequencing and how the robot moves between them greatly influence the overall performance of the application. In many such applications, the motions of the robots are still optimized manually, this being expensive and predisposing to errors. Therefore, in recent years the researchers are working to search algorithms for automatic calculation of optimal trajectories of the robot motions. This approach proved to be quite complicated, because of several factors, such as redundant kinematics, collision avoidance, ambiguous possibilities for performing the tasks, complicated objective functions, a set of possible locations for the robot base, etc., as S. Alatartsev, S. Stellmacher and F. Ortmeier [61] show. Also, it found that is difficult to compare existing approaches because they address various case studies in various industrial applications and various types of robots with different characteristics and constraints.

S. Alatartsev, starting from the finding that many kinds of tasks allow some freedom of execution, develop more studies to search a more efficient sequence of tasks. In a such study, S. Alatartsev, V. Mersheeva, M. Augustine and F. Ortmeier [62] propose a new heuristic and effective way to solve such problems by TSPN (Traveling Salesman Problem with Neighbourhoods) method, a combination of TSP (Traveling Salesman Problem) and TPP (Touring-a-sequence-of-Polygons Problem) focused only on optimizing the Euclidean distance, showing the applicability of this method.

In trying to solve an integrated problem, of tasks sequencing and path planning for a process of robotic remote laser welding, A. Kovács [63] introduces a new model, an extension of TSP method, Traveling Salesman Problem with Neighbourhoods and Duration visits (TSPND). He demonstrates through ample computational experiments, that the new approach effectively solves the relevant problems in this industry, and achieves a substantial improvement in cycle time. 
In tracking a path between two target points, the robot must usually pass by so-called via-points, placed in general to avoid certain obstacles in the workspace, or to impose a particular direction, preferred. In some cases these points must be strictly visited, in other cases, we are dealing with pseudo-via-points not intended to be strictly visited, but the passing is effected by their immediate vicinity. In this respect, H. Liu, X. Lai and W. $\mathrm{Wu}$ [64] sustain that the robot must follow exactly the path of the end effector and its laws of motion, and viapoints must be strictly visited, without any deviation, to obtain an optimal trajectory. In opposition to this opinion, S. Alatartsev, A. Belov, M. Nykolaychuk and F. Ortmeier [65] demonstrate in another study on the problem of optimizing the tasks sequencing of the industrial robots, that if it is relaxed the path through small changes in position or orientation of the endeffector, the motion law can be strictly followed, and this can lead to a significant cost reduction.

By studying several existing approaches to find a path suitable to a robot in a certain environment and their advantages and disadvantages, T. Nierhoff, S. Hirche and Y. Nakamura [66] propose a new approach to deform trajectories, while keeping their similar local shape. This new approach is based on discrete Laplace-Beltrami operator, well-known in computer graphics, but still untapped in robotics problems.

\section{Conclusion}

In the current context, the optimizations of any kind, including those in engineering, are regarded as being of particular importance, this being demonstrated by a huge number of researches, publications and scientific events dedicated to this topic. In the context of using the industrial robots on a more widely scale, and in which the optimal trajectory planning of the industrial robots proved to be a complex and difficult task, more and more researchers and specialized companies develop different theoretical and experimental studies, with different approaches to optimizing the motions of the industrial robots.

\section{References}

[1] ISO 8373:2012(en) Robots and robotic devices - Vocabulary

[2] M. W. Spong, S. Hutchinson, M. Vidyasagar, Robot modeling and control, Wiley, 2006.

[3] M. Hägele, K. Nilsson, N. Pires, R. Bischoff, "Industrial robotics", Springer Handbook of Robotics, 2nd Edition, 2016, pp. 1385-1421.

[4] H. Chen, B. Zhang, G. Zhang, "Robotic assembly", Handbook of Manufacturing Engineering and Technology, Springer, 2015, pp. 2347-2401.

[5] C. Mineo, S. G. Pierce, P. I. Nicholson, I. Cooper, "Robotic path planning for non-destructive testing - A custom MATLAB toolbox approach", Robotics and Computer-Integrated Manufacturing 37, Elsevier, 2016, pp. 1-12.

[6] R. Bloss, "Collaborative robots are rapidly providing major improvements in productivity, safety, programing ease, portability and cost while addressing many new applications", Industrial Robot: An International Journal, 43(5), 2016, pp. 463-468.

[7] R. Bogue, "Europe continues to lead the way in the collaborative robot business", Industrial Robot: An International Journal, 43(1), 2016, pp. $6-11$.
[8] B. Carlisle, "Collaborative robots: design challenges and applications", Robotic Industries Association, International Collaborative Robots Workshop, 2016.

[9] Z. Lu, C. Xu, Q. Pan, D. Xiao, F. Meng, J. Hao, "Kinematic constraint analysis in a twin-robot system for curved-surface nondestructive testing", Industrial Robot: An International Journal, 43(2), 2016, pp. 172-180

[10] O. Madsen, S. Bogh, C. Schou, R. S. Andersen, J. S. Damgaard, M. R. Pedersen, V. Krüger, "Integration of mobile manipulators in an industrial production", Industrial Robot: An International Journal, 42(1), 2015, pp. $11-18$.

[11] A. Klimchik, A. Ambiehl, S. Garnier, B. Furet, A. Pashkevich, "Comparison study of industrial robots for high-speed machining", Mechatronics and Robotics Engineering for Advanced and Intelligent Manufacturing, Part of the series Lecture Notes in Mechanical Engineering, 2016, pp. 135-149.

[12] J. Léger, J. Angeles, "Off-line programming of six-axis robots for optimum five-dimensional tasks", Mechanism and Machine Theory, 100, Springer, 2016, pp. 155-169.

[13] T. Messay, R. Ordóñez, E. Marcil, "Computationally efficient and robust kinematic calibration methodologies and their application to industrial robots", Robotics and Computer-Integrated Manufacturing, 37, 2016, pp. 33-48.

[14] G. Berselli, M. Gadaleta, A. Genovesi, M. Pellicciari, M. Peruzzini, R. Razzoli, "Engineering methods and tools enabling reconfigurable and adaptive robotic deburring", Advances on Mechanics, Design Engineering and Manufacturing, 2016, pp. 655-664.

[15] M. Halbauer, C. Lehmann, J. P. Städter, F. Leali, "Milling strategies optimized for industrial robots to machine hard materials", IEEE, 978-1-4799-0864-6/13, 2013.

[16] S. Mousavi, V. Gagnol, B. C. Bouzgarrou, P. Ray, "Model-based stability prediction of a machining robot", New Advances in Mechanisms, Mechanical Transmissions and Robotics, Mechanisms and Machine Science, vol. 46, 2016, pp. 379-387.

[17] P. Tavares, J. Lima, P. Costa, A. P. Moreira, "Multiple manipulators path planning using double A", Industrial Robot: An International Journal, 43(6), 2016, pp. 657-664.

[18] G. Boschetti, "A Picking Strategy for Circular Conveyor Tracking", Journal of Intelligent and Robotic System, 81, 2016, pp. 241-255.

[19] C. Müller, C. Weckenborg, M. Grunewald, T. S. Spengler, "Consideration of redundancies in the automated flow lines", Logistics Management, 2016, pp. 173-185.

[20] L. Wang, "Redefining the shop floor - make it as cool as a computer game", published on Sep 10, 2015, http://www.kth.se/ en/forskning/artiklar/redefining-the-shop-floor-1.590274.

[21] D. Constantinescu, E. Croft, "Smooth and time-optimal trajectory planning for industrial manipulators along specified paths", Journal of Robotic Systems, 17 (5), 2000, pp. 233-249.

[22] Paryanto, M. Brossog, M. Bornschlegl, J. Franke, "Reducing the energy consumption of industrial robots in manufacturing systems", International Journal of Advanced Manufacturing Technology, 78, 2015, pp. 1315-1328.

[23] J. Liu, W. Xu, J. Zhang, Z. Zhou, D. Pham, "Industrial cloud robotics towards sustainable manufacturing", Proceedings of the ASME 11th International Manufacturing Science and Engineering Conference, vol. 2, 2016.

[24] P. Tavares, J. Lima, P. Costa, "Double A* path planning for industrial manipulators", Advances in Intelligent Systems and Computing, 418, 2016, pp. 119-130.

[25] S. Manzoor, R. Ul Islam, A. Khalid, A. Samad, J. Iqbal, "An open-source multi-DOF articulated robotic educational platform for autonomous object manipulation", Robotics and ComputerIntegrated Manufacturing, 30, 2014, pp. 351-362.

[26] D. Spensieri, J. S. Carlson, R. Bohlin, J. Kressin, J. Shi, "Optimal robot placement for tasks execution", 6th CIRP Conference on Assembly Technologies and Systems (CATS), Procedia CIRP 44, 2016, pp. $395-400$.

[27] J. Kim, E. A. Croft, "Trajectory planning for robots: the challenges of industrial considerations, Motion Planning for Industrial Robots", IEEE International Conference on Robotics and Automation (ICRA), 2014.

[28] X. Gao, Y. Mu, Y. Gao, "Optimal trajectory planning for robotic manipulators using improved teaching-learning-based optimization algorithm", Industrial Robot: An International Journal, 43(3), 2016, pp. 308-316. 
[29] J. Kim, S. R. Kim, S. J. Kim, D. H. Kim, "A practical approach for minimum-time trajectory planning for industrial robots", Industrial Robot: An International Journal, 37(1), 2010, pp. 5161.

[30] F. Abu-Dakka, I. Assad, R. Alkhdour, M. Abderahim, "Statistical evaluation of an evolutionary algorithm for minimum time trajectory planning problem for industrial robots", International Journal of Advanced Manufacturing Technology, 2016, DOI 10.1007/s00170-016-9050-1.

[31] A. Reiter, K. Springer, H. Gattringer, A. Müller, "An explicit approach for time-optimal trajectory planning for kinematically redundant serial robots", PAMM Proceedings in Applied Mathematics and Mechanics, 15, 2015, pp. 67-68.

[32] G. Erdős, A. Kovács, J. Váncza, "Optimized joint motion planning for redundant industrial robots", CIRP Annals Manufacturing Technology, 65, 2016, pp. 451-454.

[33] F. Rubio, C. Llopis-Albert, F. Valero, J. L. Suñer, “Assembly Line Productivity Assessment by Comparing OptimizationSimulation Algorithms of Trajectory Planning for Industrial Robots", Mathematical Problems in Engineering, , Hindawi Publishing Corporation, 2015, Article ID 931048.

[34] T. Mashimo, T. Urakubo, T. Kanade, "Singularity-based four-bar linkage mechanism for impulsive torque with high energy efficiency", Journal of Mechanisms and Robotics, 7(3), 2015.

[35] D. Meike, M. Pellicciari, G. Berselli, "Energy efficient use of multirobot production lines in the automotive industry: detailed system modeling and optimization", IEEE Transactions on Automation Science and Engineering, 11(3), 2014, pp. 798-809.

[36] O. Wigstrom, B. Lennartson, A. Vergnano, C. Breitholtz, "Highlevel scheduling of energy optimal trajectories", IEEE Transactions on Automation Science and Engineering, 10(1), 2013,pp. 57-64.

[37] J. Gregory, A. Olivares, E. Staffetti, "Energy-optimal trajectory planning for robot manipulators with holonomic constraints", Systems \& Control Letters, 61(2), Elsevier, 2012, pp. 279-291.

[38] L. P. Luo, C. Yuan, R. J. Yan, Q. Yuan, J. Wu, K. S. Shin, C. S. Han, "Trajectory planning for energy minimization of industry robotic manipulators using the Lagrange interpolation method", International Journal of Precision Engineering and Manufacturing, 16(5), 2015, pp. 911-917.

[39] R. F. Fung, Y. H. Cheng, "Trajectory planning based on minimum absolute input energy for an LCD glass-handling robot", Applied Mathematical Modelling, 38, 2014, pp. 2837-2847.

[40] M. Jin, D. Wu, "Collision-free and energy-saving trajectory planning for large-scale redundant manipulator using improved PSO", Mathematical Problems in Engineering, Hindawi Publishing Corporation, Volume 2013.

[41] G. Berselli, F. Balugani, M. Pellicciari, M. Gadaleta, "Energyoptimal motions for Servo-Systems: A comparison of spline interpolants and performance indexes using a CAD-based approach", Robotics and Computer-Integrated Manufacturing, 40, Elsevier, 2016, pp 55-65.

[42] E. Oliva, G. Berselli, M. Pellicciari, A. Andrisano, "An engineering method for the power flow assessment in servoactuated automated machinery: mechatronic modeling and experimental evaluation", Robotics and Computer-Integrated Manufacturing, 38, Elsevier, 2016, pp. 31-41.

[43] M. Pellicciari, G. Berselli, F. Balugani, "On designing optimal trajectories for servo-actuated mechanisms: detailed virtual prototyping and experimental evaluation", IEEE/ASME Transactions of Mechatronics, 20(5), 2015, pp. 2039-2052.

[44] Y. Wang, K. Ueda, S. Bortoff, "A hamiltonian approach to compute an energy efficient trajectory for a servomotor system", Automatica, 49(12), 2013, pp. 3550-3561.

[45] M. Huang, Y. Hsu, R. Fung, "Minimum-energy point-to-point trajectory planning for a motor-toggle servomechanism", Transactions on Mechatronics, 17(2), 2012, pp. 337-344.

[46] H. I. Lin, "A fast and unified method to find a minimum-jerk robot joint trajectory using particle swarm optimization", Journal of Intelligent and Robotic System, 75, 2014, pp. 379-392.

[47] Z. Miljković, M. Petrović, "Application of modified multiobjective particle swarm optimisation algorithm for flexible process planning problem", International Journal of Computer Integrated Manufacturing, 30(2-3), 2017, pp. 271-291.

[48] D. Gleeson, S. Bjorkenstam, R. Bohlin, J. S. Carlson, B. Lennartson, "Towards energy optimization using trajectory smoothing and automatic code generation for robotic assembly", 6th Conference on Assembly Technologies and Syatems (CATS), Procedia CIRP, vol. 44, 2016, pp. 341-346.

[49] H. Liu, X. Lai, W. Wu, "Time-optimal and jerk-continuous trajectory planning for robot manipulators with kinematic constraints", Robotics and Computer-Integrated Manufacturing, 29(2), 2013, pp. 309-317.

[50] S. Perumaal, N. Jawahar, "Synchronized trigonometric curve trajectory for jerk-bounded time-optimal pick and place operation", International Journal of Robotics and Automation, 27(4), 2012, pp. 385-395.

[51] A. Gasparetto, V. Zanotto, "Optimal trajectory planning for industrial robots", Advances in Engineering Software, 41, 2010, pp. 548-556.

[52] A. Gasparetto, A. Lanzutti, R. Vidoni, V. Zanotto, "Experimental validation and comparative analysis of optimal time-jerk algorithms for trajectory planning", Robotics and ComputerIntegrated Manufacturing, 28(2), 2012, pp. 164-181.

[53] F. Liu, F. Lin, "Time-jerk optimal planning of industrial robot trajectories", International Journal of Robotics and Automation, 31(1), 2016, pp. 1-7.

[54] H. Duan, R. Zhang, F. Yu, J. Gao, Y. Chen, "Optimal trajectory planning for glass-handing robot based on execution time, acceleration and jerk", Journal of Robotics, Hindawi Publishing Corporation, 2016.

[55] H. Esfandiar, M. H. Korayem, "Optimal point to point path planning of flexible manipulator under large deformation by using harmony search method", Journal of Theoretical and Applied Mechanics, 54(1), 2016, pp. 179-193.

[56] M. Jafari, A. Shahmansoorian, "PI/Backstepping control of snake robot optimazed by genetic algorithm", IEEE 978-1-4799-30109/14/, CCECE, Toronto, Canada, 2014.

[57] P. Costa, J. Lima, A. I. Pereira, P. Costa, A. Pinto, "An optimization approach for the inverse kinematics of a highly redundant robot", Advances in Intelligent Systems and Computing, 427, Springer, 2016, pp. 433-442.

[58] F. Anton, S. Anton, S. Răileanu, T. Borangiu, "Optimizing trajectory points for high speed robot assembly operations", Advances in Robot Design and Intelligent Control, 371, 2016, pp. 127-135.

[59] W. Xiao, J. Huan, "Redundancy and optimization of a 6R robot for five-axis milling applications: singularity, joint limits and collision”, Production Engineering, 6(3), 2012, pp. 287-296.

[60] S. S. Chiddarwar, N. R. Babu, "Optimal trajectory planning for industrial robot along a specified path with payload constraint using trigonometric splines", International Journal of Automation and Control, 6(1), 2012.

[61] S. Alatartsev, S. Stellmacher, F. Ortmeier, "Robotic Task Sequencing Problem: A Survey", Journal of Intelligent and Robotic System, 80, 2015, pp. 279-298.

[62] S. Alatartsev, V. Mersheeva, M. Augustine, F. Ortmeier, "On Optimizing a Sequence of Robotic Tasks", IEEE/RSJ International Conference on Intelligent Robots and Systems (IROS), November 3-7, 2013. Tokyo, Japan.

[63] A. Kovács, "Integrated task sequencing and path planning for robotic remote laser welding", International Journal of Production Research, 54(4), 2016, pp. 1210-1224.

[64] H. Liu, X. Lai, W. Wu, "Time-optimal and jerk-continuous trajectory planning for robot manipulators with kinematic constraints", Robotics and Computer-Integrated Manufacturing, 29(2), 2013, pp. 309-317.

[65] S. Alatartsev, A. Belov, M. Nykolaychuk, F. Ortmeier, "Robot trajectory optimization for the relaxed end-effector path", Informatics in Control, Automation and Robotics (ICINCO), Vienna, Austria, 2014.

[66] T. Nierhoff, S. Hirche, Y. Nakamura, "Spatial adaption of robot trajectories based on laplacian trajectory editing", Autonomous Robots, 40, Springer, 2016, pp. 159-173. 